\title{
Satisfacción de usuarios en consulta externa de hospitales públicos de la región Ica, 2018.
}

\author{
Quality of service in external consultation of public hospitals of the \\ Ica region, 2018.
}

Becerra-Canales Bladimiri, , a.

1. Dirección de Investigación y Producción Intelectual. Universidad Privada de Ica. Perú.

2. Dirección Regional de Salud Ica. Ministerio de Salud, Ica-Perú.

a. Doctor en Salud Publica

\section{Correspondencia:}

Bladimir Becerra-Canales

Número de celular: 956690060

Correo Electrónico:

icapredica@gmail.com

Domicilio: CC.HH La Angostura III Etapa H-10, Ica Perú.

Contribuciones De Autoría: BBC: Contribuciones en la concepción y diseño del manuscrito, recolección, análisis e interpretación de los datos, redacción y revisión crítica del contenido del manuscrito y aprobación final del artículo.

Conflicto De Intereses: No declarados.

Financiamiento: Autofinanciado.

\section{Como Citar}

Becerra-Canales

Bladimir.

Satisfacción de usuarios en consulta externa de hospitales públicos de la región Ica, 2018. Rev méd panacea 2018; (3): 89-92

\section{RESUMEN}

Objetivo: Conocer el grado de satisfacción de los usuarios externos que acuden a la consulta externa en seis hospitales públicos de la Región Ica, Mayo del 2018. Materiales y métodos: Estudio observacional, prospectivo, trasversal y de nivel descriptivo, la muestra estuvo representada por 1254 usuarios externos, que fueron seleccionados por muestreo probabilístico, estratificado y sistemático, en 6 hospitales de la Región, mediante una medición cruzada. Resultados: El grado de satisfacción de los usuarios externos es del 55.8\% en el Hospital Regional de Ica (HRI), 80.2\% Hospital Santa María del Socorro de Ica (HSMSI), 71.9\% Hospital San Juan de Dios de Pisco (HSJDP), 69.7\% Hospital San José de Chincha (HSJCH), 72.3\% Hospital de Apoyo de Palpa (HAP) y 81.6\% Hospital Apoyo de Nazca (HAN). Conclusiones: Existen diferencias significativas entre los grados de satisfacción de los usuarios externos en las instituciones hospitalarias de la Región Ica ( $p$-valor = 0.0234).

Palabras clave: Satisfaccion de usuarios, consulta externa. (fuente: DeCS BIREME).

\begin{abstract}
Objective: To know the degree of satisfaction of external users who attend the outpatient clinic in six public hospitals of the Ica Region, May 2018. Materials and methods: Observational, prospective, cross-sectional and descriptive level study, the sample was represented by 1254 external users, who were selected by probabilistic, stratified and systematic sampling, in 6 hospitals of the Region, by means of a cross measurement. Results: The degree of satisfaction of external users is $55.8 \%$ en el Hospital Regional de Ica (HRI), $80.2 \%$ Hospital Santa María del Socorro de Ica (HSMSI), 71.9\% Hospital San Juan de Dios de Pisco (HSJDP), 69.7\% Hospital San José de Chincha (HSJCH), 72.3\% Hospital de Apoyo de Palpa (HAP) y 81.6\% Hospital Apoyo de Nazca (HAN). Conclusions: There are significant differences between the levels of satisfaction of external users in the hospital institutions of the Ica Region ( $p$-value $=0.0234$ ).
\end{abstract}

Keywords: User satisfaction, external consultation. (source: DeCS BIREME). 


\section{INTRODUCCIÓN}

La calidad en la prestación de los servicios de salud es sin duda una preocupación medular en la gestión del sector salud, no sólo por la aspiración a la mejora de las capacidades institucionales, sino sobre todo porque en el eje de todos los procesos se encuentra la vida de las personas, cuya salud debe ser promovida y protegida como obligación del Estado. La calidad en la prestación es condición de eficacia de los esfuerzos e intervenciones desplegadas y garantía de seguridad y trato humano para todos y cada uno de los usuarios 1.

Cabe resaltar que durante los últimos años se han desarrollado grandes esfuerzos y múltiples iniciativas para la medición de la calidad a través de la evaluación de la satisfacción de los usuarios externos; ante la gran demanda de atención en los Establecimientos de Salud se percibe un grado de insatisfacción creciente de los usuarios del sistema de salud de allí la necesidad de explorar otras metodologías con cuestionarios sencillos y de fácil aplicación que muestren resultados para la toma de decisiones y genere un proceso de mejora continua de la calidad de atención2.

En ese contexto rutinariamente se evalúa la satisfacción de los usuarios en los servicios de salud, con el fin de conocer las causas de su insatisfacción y así tomar medidas que permitan mejorar la percepción de los usuarios e incrementar los niveles de satisfacción de nuestros pacientes, quienes constituyen la razón de ser de los establecimientos sanitarios y del personal que labora en ella.

Sin embargo, a pesar de los esfuerzos desplegados, la insatisfacción es una constante común en la consulta externa de los hospitales públicos en nuestra región, la percepción y expectativas del servicio de salud es dinámico y cambiante, en ese contexto, la satisfacción usuaria constituye un elemento particularmente importante para la evaluación de los servicios de salud. En primer orden, la satisfacción de los usuarios es el resultado final del sistema sanitario, en segundo orden, contribuye a mejorar la calidad de la atención, en función a los planes de mejora que se deriven de los resultados obtenidos de su medición. Por lo tanto, resulta fundamental evaluar la satisfacción de los usuarios externos en los servicios de salud de las instituciones sanitarias.

\section{MATERIALES Y MÉTODOS}

Se realizó un estudio de nivel descriptivo, de tipo observacional, prospectivo y transversal, el tamaño de la muestra estuvo representada por 1254 usuarios externos (HRI 380, HSMSI 216, HSJDP 167, HSJCH 261, HAN 147 y HAP 83 usuarios externos), las unidades muéstrales fueron seleccionadas mediante procedimiento probabilístico estratificado sistemático. Para la recolección de la información se utilizó la técnica de entrevista y como instrumento una escala tipo Likert, el mismo que fue sometido a procedimientos de validación por juicio de expertos y prueba piloto. El área de estudio fueron 06 instituciones hospitalarias de la Región Ica, se tomó en cuenta aspectos éticos y los datos fueron recolectados durante el mes de Mayo del año 2018, mediante una medición cruzada a cargo de un equipo de encuestadores debidamente capacitados. La base de datos fue sometido a un análisis estadístico, en la Oficina de Gestión de la Calidad de la Dirección Regional de Salud Ica, utilizando el paquete estadístico Statistical Package for the Social Sciences (SPSS) para Windows versión 21.0 en Español.

\section{RESULTADOS:}

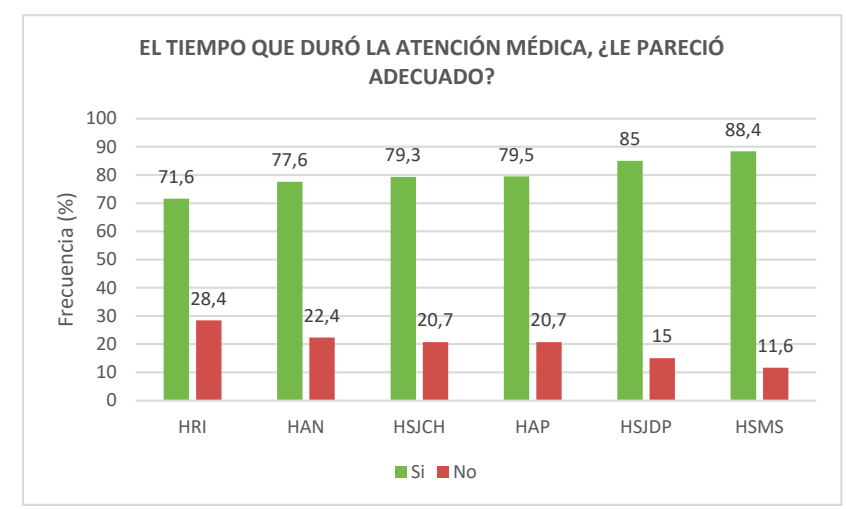

Gráfico Nº1: Percepción del tiempo que duro la atención médica.

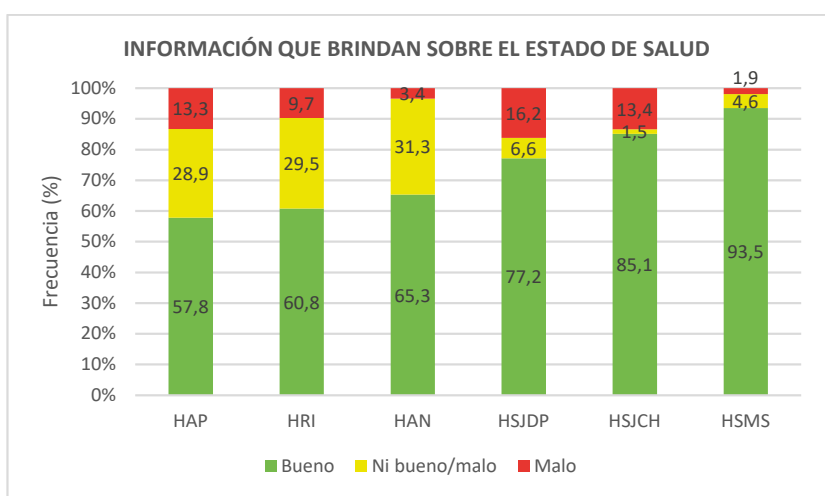

Gráfico N 02: Calificación de los usuarios externo, respecto a la información que brindan los profesionales, sobre el estado de salud.

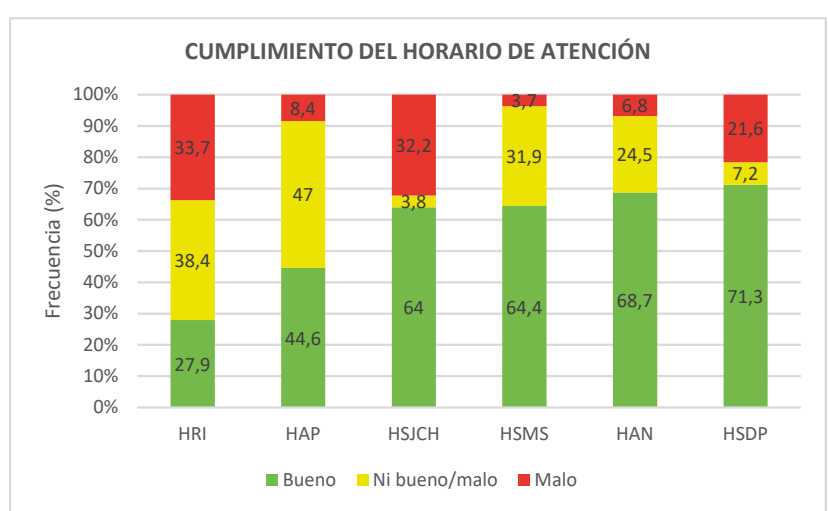

Gráfico N 03: Calificación de los usuarios externo, respecto al cumplimiento del horario de atención del personal de salud. 


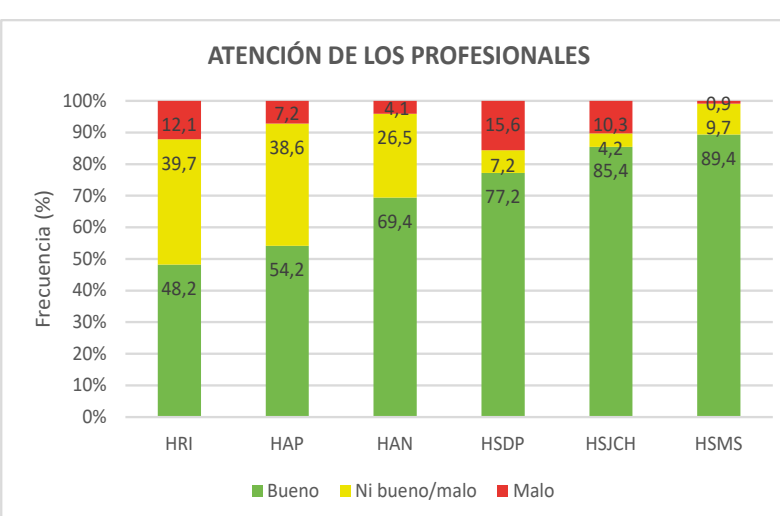

Gráfico No 04: Calificación de los usuarios externo, respecto a la atención de los profesionales.

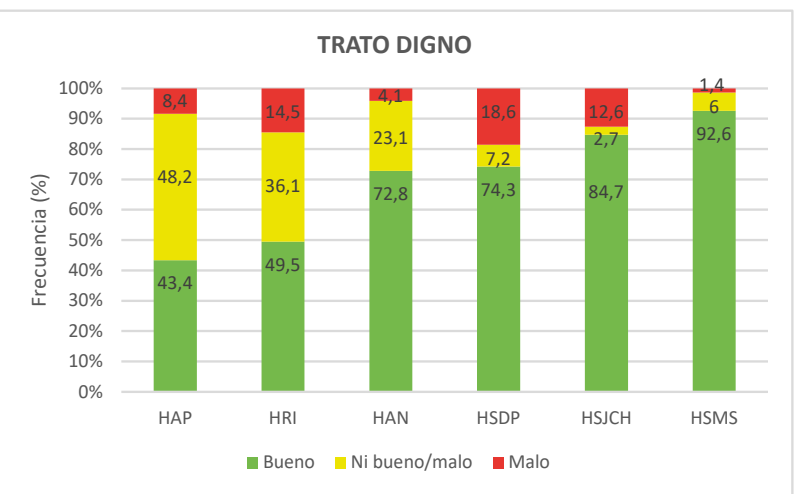

Gráfico Nº5: Calificación de los usuarios externo, respecto al trato digno.

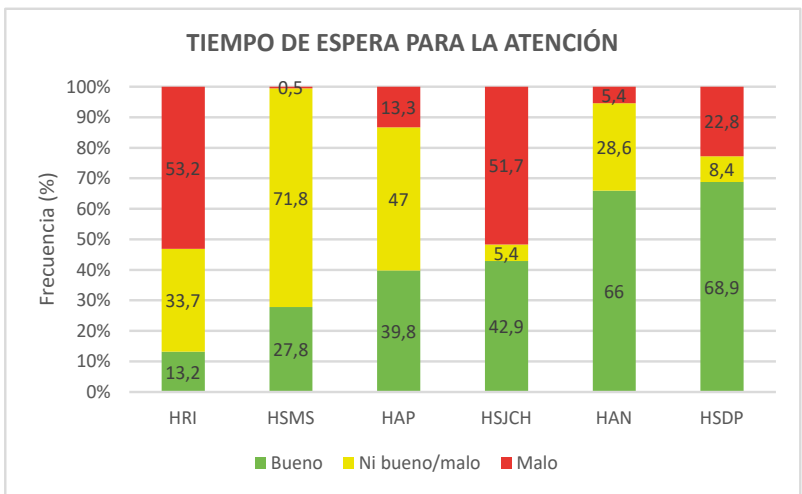

Gráfico N 06: Calificación de los usuarios externo, respecto al tiempo de espera para la atención.

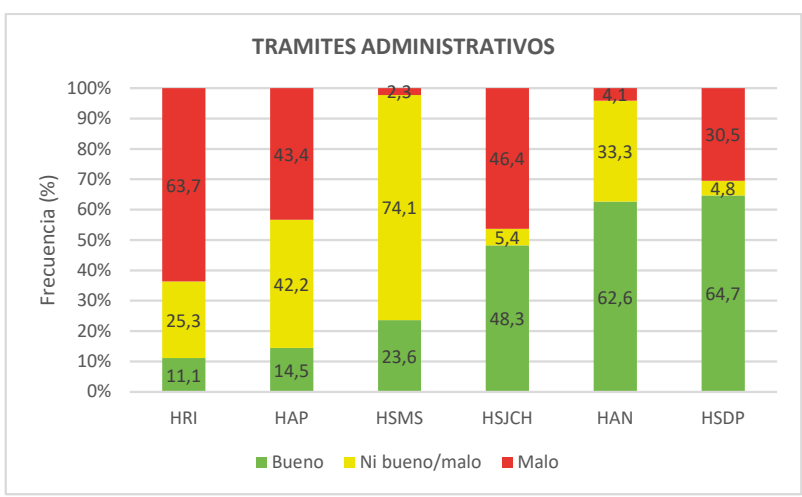

Gráfico Nº7: Calificación de los usuarios externo, respecto a los trámites administrativos.
NIVEL DE SATISFACCION DE LOS USUARIOS EXTERNOS SEGUN HOSPITAL. (05/2018)

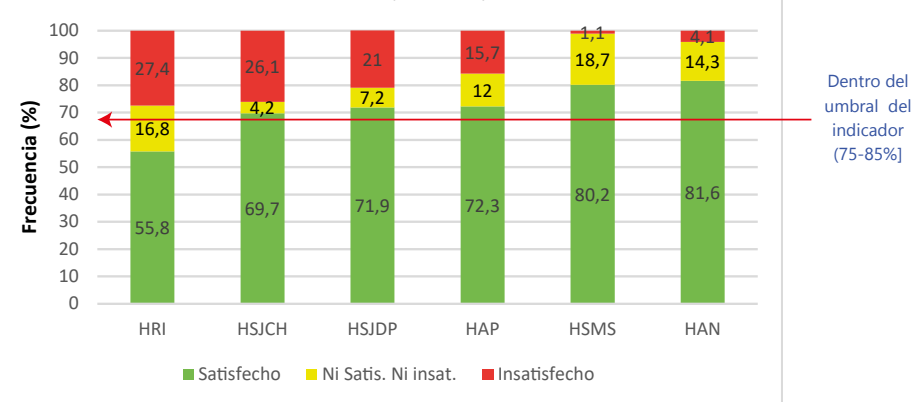

Gráfico $\mathbf{N}^{\circ}$ 08: Nivel de satisfacción de los usuarios externos según institución hospitalaria de la región Ica.

\section{DISCUSIÓN:}

La distribución de los grados de satisfacción de los usuarios externos en las instituciones hospitalarias de la Región Ica, es heterogéneo; con diferencias significativas de estos grados de satisfacción según instituciones hospitalaria ( $p$-valor $=0.0234$ ). El grado de satisfacción más bajo se encontró en el Hospital Regional de Ica (55.8\%), y el más alto en el Hospital de Apoyo de Nasca (81.6\%); la distribución de los grados de satisfacción no es homogénea en los hospitales de la región Ica, correspondiéndose con los hallados en diversos Hospitales del Perú, tales como el Hospital Nacional Arzobispo Loayza, donde se encontró un $44.36 \%$ de satisfacción3; Hospital Docente Madre-Niño "San Bartolomé" donde la satisfacción fue de 58.1\%4; el Hospital Regional de Lambayeque donde se halló una insatisfacción global algo más elevada que en nuestros hospitales, 89,80\%5; Asimismo, en Hospitales del seguro social - EsSalud como el Hospital Edgardo Rebagliati Martins, donde la media se satisfacción fue del 65.6\%6 y el Hospital Naylamp Es Salud-Chiclayo 59\% de satisfaccion7; dentro del sector castrense, encontramos que en el Hospital Nacional PNP "Luis N. Sáenz" el nivel de satisfacción fue 26.9\%8; Hospital Regional Policía Nacional del Perú-Chiclayo, 76.8\%9; y finalmente el Hospital Nacional Almanzor Aguinaga, reporto 60\% de satisfacción10; estos hallazgos demuestran que en el país, la insatisfacción usuaria, es una constante común en la consulta externa hospitalaria.

En efecto el tiempo de espera prolongado, los trámites administrativos burocráticos y el incumplimiento del horario de atención por parte del personal de salud, constituyen las principales causas de insatisfacción de los usuarios externos en los hospitales de la región Ica, por ello se hace necesario que los gestores sanitarios tomen en cuenta estas dimensiones de la calidad de atención, para la mejora de los procesos.

Los resultados encontrados en el presente estudio, plantean la necesidad de realizar mediciones periódicas e investigaciones de nivel relacional, explicativo y aplicativo sobre la línea de investigación en cuestión. Se propone además como solución al problema 
implementar acciones de mejora $y / o$ proyectos de mejora continua en la gestión de historias clínicas, gestión de recursos humanos, disminución de los tiempos de espera entre otros, que permitan superar de manera priorizada la problemática encontrada y que

\section{REFERENCIAS BIBLIOGRÁFICAS}

1. Ministerio de Salud. Sistema de Gestión de la Calidad. Dirección General de las Personas. Dirección Ejecutiva de la Calidad en Salud. Norma Técnica: R.M. 519-2006/MINSA. 2006. p.6.

2. Ministerio de Salud. Guía técnica y aplicativo para la evaluación de la satisfacción del usuario externo en los establecimientos de salud y servicios médicos de apoyo. RM N²527/MINSA.2011.p 11.

3. Casalino-Carpio G. Calidad de servicio de la consulta externa de Medicina Interna de un hospital general de Lima mediante la encuesta Servqual. Rev Soc Peru Med Interna [Internet]. 2008 [Citado 9/10/18]; vol 21 (4): pag 143-152. Recuperado a partir de: http://sisbib.unmsm.edu.pe/bvrevistas/spmi/v21n4/p df/a03v21n4.pdf

4. Vásquez W, Arimborgo C, Pillhuaman N, Vallenas G. Satisfacción del usuario de consulta externa en el Hospital Nacional Docente Madre Niño San Bartolomé. investigaciones sociales [Internet]. 2009 [Citado 9/10/18]; Vol.13 (22), pp.337-353. Recuperado a partir de: http://www.acuedi.org/ddata/3351.pdf

5. Niño-Effio B, Perales JC, Chavesta X. Calidad de servicio en la consulta externa de un hospital de la región Lambayeque octubre 2010. Rev. cuerpo méd. [Internet]. 2012 [Citado 9/10/18]; Vol.5 (1), pp.5-9. Recuperado a partir de: Users/USUARIO/Downloads/Dialnet-CalidadDeServici oEnLaConsultaExternaDeUnHospitalDe-4061256.pdf

6. Acuña-Zavala PD, Adrianzén R SB, Almeyda Alvarado $K A$, Aguilar J. Evaluación del nivel de satisfacción del adulto entre 60 a 90 años en la consulta ambulatoria del Hospital Edgardo Rebagliati Martins. Horizonte influye negativamente en la satisfacción de los usuarios externos que acuden a los servicios de salud de los hospitales públicos; nuevas mediciones de la satisfacción usuaria deberán ser realizadas para evaluar el impacto de las soluciones planteadas.

Médico [Internet]. 2012 [Citado 9/10/18]; Vol.12 (3), pp.22-26. Recuperado a partir de: http://www.redalyc.org/pdf/3716/371637126004.pd f

7. López J. Calidad de atención médica y satisfacción del usuario atendido en la consulta externa del Hospital Naylamp EsSalud - Chiclayo. [En línea]. (2011) Disponible en URL: http://www.buenastareas.com/ensayos/Satisfaccion -De-Usuario-y-Calidad-De/1592529.html

8. Tinoco M. Satisfacción del usuario externo en el servicio de consulta externa del Hospital Nacional P.N.P. Luis Nicasio Sáenz, 2015. [Tesis en Internet]. [Lima-Perú]. 2016 [Citado 9/10/18]. Recuperado a partir de: http://cybertesis.urp.edu.pe/handle/urp/473

9. León A. Niveles de satisfacción y calidad de atención médica de los usuarios del servicio de consulta externa en el Hospital Regional Policía Nacional del Perú, Chiclayo, 2016. [Tesis en Internet]. [Lambayeque-Perú]. 2016 [Citado 9/10/18]. Recuperado a partir de: http://repositorio.unprg.edu.pe/bitstream/handle/U NPRG/1478/BC-TES-TMP-315.pdf? sequence=1\&isA llowed=y

10. Mego F. Nivel de satisfacción del usuario de los servicios de consulta externa del Hospital Nacional Almanzor Aguinaga Asenjo ESSALUD. [Tesis en Internet]. [Lima-Perú]. 2013 [Citado 9/10/18]. Recuperado a partir de: http://www.repositorioacademico.usmp.edu.pe/bitst ream/usmp/2213/3/mego_fn.pdf

\section{Las ediciones anteriores de revista médica PANACEA están disponibles en:}

\section{www.revpanacea.unica.edu.pe}

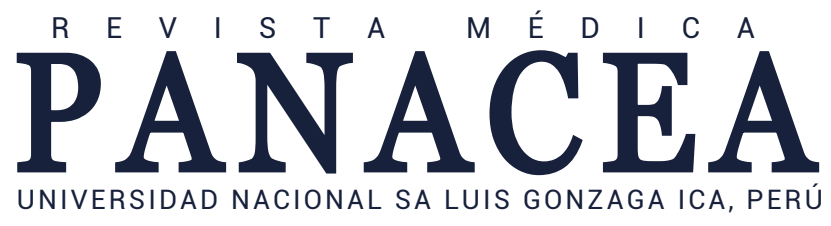

